\title{
Impact of Constraints on the Complexity and Performance of Channel Assignment in Multi-Hop Wireless Networks
}

\author{
Chetan Nanjunda Mathur, M.A. Haleem, K.P. Subbalakshmi and \\ R. Chandramouli
}

Department of Electrical and Computer Engineering, Stevens Institute of

Technology, Hoboken, NJ 07030, USA; e-mail: mouli@stevens.edu

\begin{abstract}
In this paper we systematically study several channel assignment problems in multi-hop ad-hoc wireless networks in the presence of several constraints. Both regular grids and random topology models are considered in the analysis. We identify three fairness constraints (unfair, fair, and 1-fair), Signal to Interference Ratio (SINR) constraint (to measure the link quality) and balance constraint (for uniform assignment) and study their impact on the complexity of the channel assignment problems. Note that these constraints have an impact on the network capacity, lifetime and connectivity.

Although optimal channel assignment for links in a multi-hop wireless network has been shown to be NP complete, the impact of fairness, link quality and balance constraints on the hardness of channel assignment problems is not well studied. In this paper, we show that a class of unfair SINR constrained channel assignment problems can be solved in polynomial time. We show that when fairness is desired the channel assignment problems are NP Complete. We propose two heuristic algorithms that provide 1-fair and fair channel assignments, comment on their complexity and compare their performance with optimal solutions.
\end{abstract}

Keywords: scheduling, set covering, graph coloring, wireless ad-hoc networks, NP.

Journal of Cyber Security and Mobility, Vol. 1, 161-187.

(c) 2012 River Publishers. All rights reserved. 


\section{Introduction}

Based on the number of wireless hops, wireless networks can be classified into last-hop and multi-hop networks. Last hop networks refer to wired networks whose last hop is wireless. These types of networks are also called infrastructure networks, the cellular network being an example. On the other hand if all the hops of a wireless network are comprised of wireless links, the network is called a multi-hop wireless network. An example for such networks is the ad-hoc network. In this paper we concentrate on multi-hop wireless networks.

As all the nodes in a multi-hop network share a common wireless communication medium, some type of coordination is desired to avoid collisions or simultaneous channel access by two or more devices. Most of the literature on medium sharing techniques can be classified into two types: random access techniques and channel assignment techniques. Random access techniques, as the name suggests, use some type of randomized protocols to access the channel in such a way that collisions are minimized. Examples of random access techniques include the well known Aloha protocol [11] and the Carrier Sense Multiple Access with Collision Avoidance (CSMA/CA). Channel assignment techniques on the other hand, use orthogonal sub-channels as the key to achieving simultaneous communication and mitigate mutual interference. This is used both in networks with global co-ordination and distributed co-ordination. The channel assignment techniques in wide use are Time Division Multiple Access (TDMA), Frequency Division Multiple Access (FDMA), Code Division Multiple Access (CDMA), Orthogonal Frequency Division Multiplexing (OFDM) and some combinations of these [11]. Therefore by the term channel we mean, a time frame in TDMA, a segment of allocated spectrum in FDMA, or a collection of orthogonal codes in CDMA. The term sub-channel thus implies time slots of a time frame, frequency channels within the segment of the spectrum or a code in the collection of orthogonal codes. For the analysis in the paper we assume time slot assignment.

Depending on what is being scheduled, channel assignment can be of two types. When nodes are assigned to different sub-channels [4, 7, 10,14], it is referred to as node scheduling/assignment. Node scheduling is usually referred to as broadcast scheduling. Similarly, link scheduling is when each of the links in the network are assigned sub-channels. Link scheduling is also referred to as point to point scheduling $[1,2,6,12]$. Although node and link scheduling problems differ in terms of number of elements to be scheduled 
Table 1 Notations used.

\begin{tabular}{ll}
\hline$V$ & Set of all nodes (vertices of G) \\
$e_{i, j}$ & Link formed when node $i$ transmits to node $j$ \\
& Note that the existence of $e_{i, j}$ does not imply $e_{j, i}$ \\
$E$ & Set of all possible links in the network (edges in G) \\
$E_{t}$ & Set of links that are active at time $t$, where $t>0$ \\
$S I R\left(e_{i, j}, t\right)$ & Signal to interference ratio of edge $e_{i, j}$ at time $t$ \\
$T$ & Total number of active sub-channels (time-slots) \\
$S_{i}$ & Set of sub-channels assigned to link $e_{i}$ \\
\hline
\end{tabular}

and computational complexity, in general a solution to link scheduling can be transformed to node scheduling by transforming the nodes in the given graph into links and by applying link scheduling on it. Some of the work that propose algorithms to achieve both broadcast scheduling and point to point scheduling are $[13,16]$. In our current work we only consider link scheduling problems. Hence in the rest of the paper by channel assignment we refer to link scheduling. We identify three constraints in channel assignment: (a) signal to interference noise ratio constraint, (b) fairness constraint, and (c) balance constraint. A formal definition of these constraints are given in later sections. We note that these constraints impact the network capacity, connectivity, lifetime and traffic load. In addition to these we also discuss atomic constraints that are dictated by the physical layer of the nodes.

\section{Network Model}

To formulate the channel assignment problem in a multi-hop wireless network, we map a given wireless network to a graph $G=(V, E)$, where the vertices, $V$, represent the wireless devices (nodes) and the edges, $E$, represent the transmission links between these devices. We capture the multi hop wireless networks that occur in practice using graphs as described later in this section. The network graphs used in the experimental section are generated using this model. Some of the notations used in the rest of the paper are summarized in Table 1.

\subsection{Random Network Model}

In this case, the nodes are randomly placed in a given network area. A fixed power level is allocated to each of the nodes. We define the parameter $R_{\max }$ of the network as the length of the longest active edge. Similarly $R_{\min }$ is the 


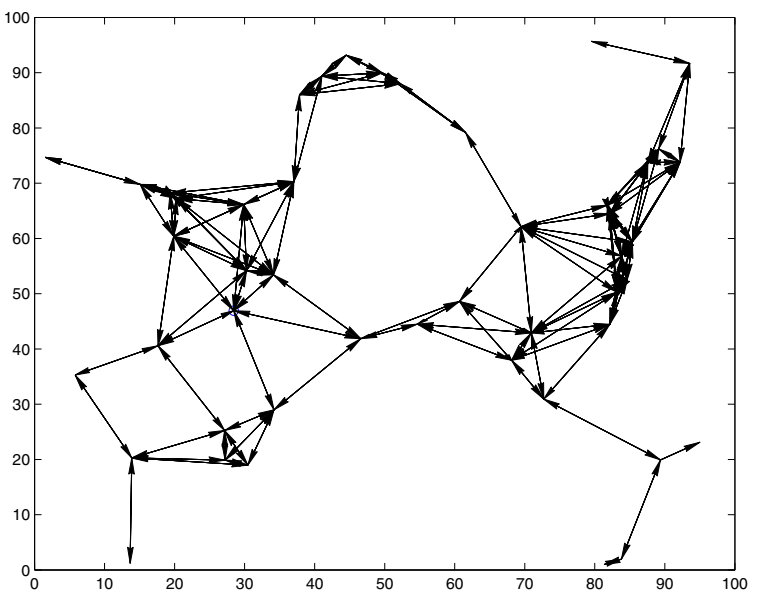

Figure 1 Example of random network with area $A=100 \times 100$ square meters, 49 nodes and range $R_{\max }=20$ meters, $R_{\min }=1.362$ meters.

length of the smallest active edge in the network. An example of random network model is shown in Figure 1.

\subsection{Grid Based Network Model}

In the grid based network model we assume that the nodes are placed on a rectangular grid. In this model all the edges formed are of same length and all transmitting nodes have same range. Hence the maximum transmission range $R_{\max }$ is same as the minimum transmission range $R_{\min }$. An example of grid based network model for a 49 node network is shown in Figure 2. This type of model is also used in, for example, [5].

\section{Channel Assignment Constraints}

\subsection{Atomic Constraint}

An atomic constraint is a symmetric relationship between two vertices or two edges in a graph [13]. Two vertices/edges that are mutually constrained cannot be scheduled in the same sub-channel. In [13], the author identifies atomic constraints and models scheduling/assignment problems as a constraint set, $A_{c}$, over the atomic constraints. For the sake of clarity, in Table 2 we summarize the 3 atomic link constraints that are relevant to our work. 


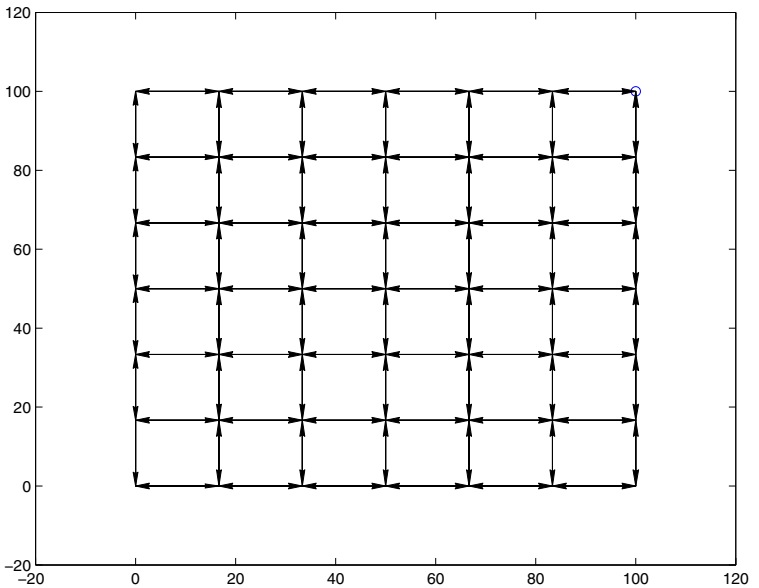

Figure 2 Example of grid network with area $A=100 \times 100$ square meters, 49 nodes and range $R_{\min }=R_{\max }=20$ meters.

Table 2 Atomic Link Constraints

\begin{tabular}{lc}
\hline Constraint & Implication \\
\hline$(E, 0, t t)$ & $\nexists e_{i, j}, e_{i, k}$ s.t. $\left\{e_{i, j}, e_{i, k}\right\} \subseteq E_{t}, t \in\{1, \ldots, T\}$ \\
$(E, 0, r r)$ & $\nexists e_{j, i}, e_{k, i}$ s.t. $\left\{e_{j, i}, e_{k, i}\right\} \subseteq E_{t}, t \in\{1, \ldots, T\}$ \\
$(E, 0, t r)$ & $\nexists e_{i, j}, e_{k, i}$ s.t. $\left\{e_{i, j}, e_{k, i}\right\} \subseteq E_{t}, t \in\{1, \ldots, T\}$ \\
\hline
\end{tabular}

For example the TDMA link scheduling/assignment problem can be modeled as a constraint set $A_{c}(T D M A)=\{(E, 0, t t),(E, 0, r r)\} .(E, 0, t t)$ means that in any time slot a node cannot perform multiple simultaneous direct transmissions to more than one receiving node. $(E, 0, r r)$ means that a receiving node cannot directly receive simultaneously from two different transmitting nodes in a given time slot.

\subsection{SINR Constraint}

Although link schedules that satisfy atomic constraints avoid collisions in the wireless network, they do not impose any kind of guarantee on the quality of received signal at each receiver. To assure a minimum quality at each receiver in the network, we define a constraint on the signal to interference plus noise ratio (SINR). The SINR constraint simply says that the assignment should guarantee a desired minimum SINR requirement (say $\gamma_{t h}$ ) for all the links assigned in all of its sub-channels. While the optimal assignment of links and power control to satisfy this constraint requires the consideration of the 
"physical model" of the system, a simplified analysis can be carried out with a conservative approach leading to the so called "protocol model" [5]. We now define the SINR constraint in the physical model which is then simplified to distance constraint in the protocol model.

Let $E_{t}$ be the set of all links assigned to the sub-channel $t$, then under the physical model the scheduling/assignment is said to satisfy the minimum SINR constraint if

$$
\operatorname{SINR}\left(e_{i j}, t\right) \geq \gamma_{t h}, \forall t \in\{1, \ldots, T\}, \forall e_{i j} \in E_{t}
$$

\subsection{Distance Constraint}

While the physical model simply states the constraint, the protocol model discussed below provides a simplified method to impose the constraint.

If link $e_{i}$ is assigned sub-channel $t$ (Figure 3) i.e., $e_{i} \in E_{t}$, then it is said to satisfy the minimum distance ratio constraint [5], if $\forall e_{j} \in E_{t}-e_{i}$,

$$
d\left(\operatorname{Tx}\left(e_{j}\right), R x\left(e_{i}\right)\right) \geq\left(1+\delta_{t h}\right) R_{\max },
$$

where $R_{\max }$ is the maximum transmission radius in the network, $T x\left(e_{j}\right)$ denotes the transmitter of link $e_{j}$ and $R x\left(e_{i}\right)$ is the receiver of link $e_{i}$. That is, the distance between the receiver of the given link and the transmitters of other active links sharing the same sub-channel $t$ should be larger by a factor of $1+\delta_{t h}$ compared to the maximum transmission radius of the network. Note that for the grid based network model $R_{\max }=R_{\min }=R$.

\subsection{Fairness}

Depending on how many sub-channels each link is assigned to, channel assignment can be classified as unfair, 1-fair and fair. A channel assignment is called unfair if there is at least one link that is not assigned to any of the sub-channels. This leads to the following definition.

Definition 1 Let $S_{i}$ be the set of sub-channels assigned to link $e_{i}$, then $S_{i}$ is represented as

$$
S_{i}=\left\{t, e_{i} \in E_{t} \forall t \in\{1, \ldots, T\}\right\}
$$

An assignment is said to be unfair if

$$
\exists i \in\{1, \ldots,|E|\} \text { s.t. }\left|S_{i}\right|=0
$$




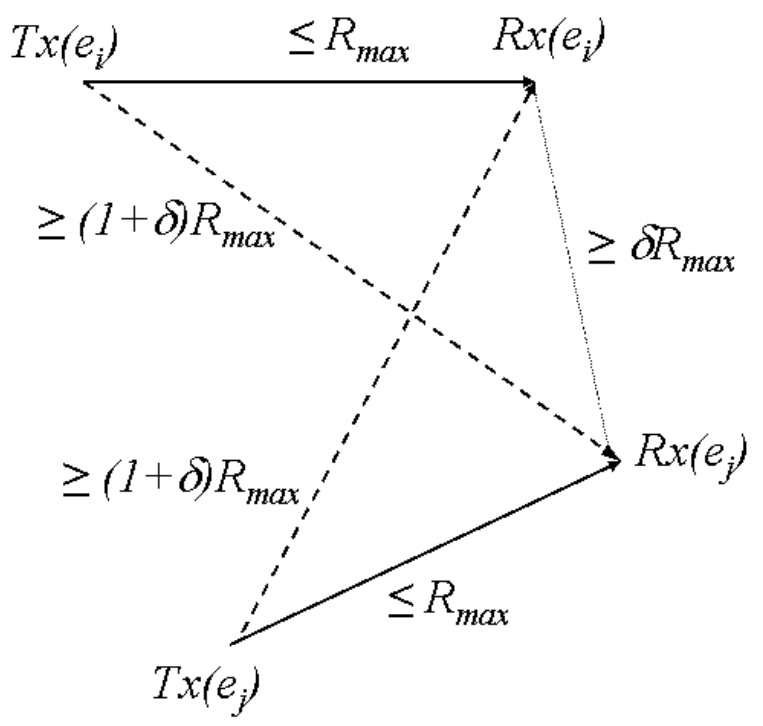

Figure 3 Pairwise distance constraints between two given links $e_{i}$ and $e_{j}$ in a wireless network, with distance ratio constraint of $\delta$.

Under such a channel assignment the node degree will reduce and the connectivity of the graph may be lost, thus making routing between some source destination pairs impossible. Nevertheless, a network that prefers "maximum overall throughput" regardless of fairness may adopt an unfair policy.

In order to preserve the connectivity of the underlying communication graph, we need every link to be assigned to at least one sub-channel. This gives rise to the following definition of 1-fairness.

Definition 2 A channel assignment is said to satisfy the 1-fairness constraint if

$$
\left|S_{i}\right| \geq 1, \forall i \in\{1, \ldots,|E|\}
$$

Although 1-fair assignment preserves the connectivity of the graph it can be biased. Some of the links could be assigned more sub-channels. Hence, when every link is assigned to exactly same number of sub-channels, such an assignment achieves fairness.

Definition 3 A channel assignment is said to be fair if,

$$
\left|S_{i}\right|=d, \forall i \in\{1, . .,|E|\}
$$


where $d \geq 1$ is a constant.

\subsection{Balance}

Unfair, 1-fair and fair assignments need not be balanced over sub-channels; some of the sub-channels may be assigned more number of links than the others. To achieve an ideally balanced assignment, it is necessary that every sub-channel contains exactly the same number of active links. That is,

$$
\left|E_{t_{1}}\right|=\left|E_{t_{2}}\right|, \forall t_{1}, t_{2} \in\{1, \ldots, T\}
$$

otherwise, the channel assignment is said to be unbalanced. Balanced assignment will help in maintaining an acceptable level of load per time slot.

It is easy to see that ideally balanced assignment is not always feasible; one example is when the number of links in the network is prime. Hence, we define a channel assignment to be $\epsilon_{t h}$ unbalanced if the maximum absolute difference in the number of active links between two sub-channels is not greater than $\epsilon_{t h}$. That is,

$$
\max \left(a b s\left(\left|E_{t_{1}}\right|-\left|E_{t_{2}}\right|\right)\right) \leq \epsilon_{t h}, t_{1}, t_{2} \in\{1, \ldots, T\}
$$

Note that $\epsilon_{t h}$ is upper bounded by $|E|$.

\section{Link Scheduling/Sub-Channel Assignment Problems}

Definition 4 A Channel Assignment Problem (CAP) is represented as a twotuple $(\mathfrak{G}, \mathfrak{C})$, where

1. $\mathfrak{G}$ is a two-tuple $(\mathbb{G}, \mathbb{P})$ representing the wireless network with,

- $\mathbb{G}$ being a set of graph representations $G$ of the wireless network.

- $\mathbb{P}$ being a set of network parameters such as geographical area $A$, set of link gains $\{h\}$ as well as the presencelabsence of "power control".

2. $\mathfrak{C}$ is a four-tuple $(\mathbb{A}, \mathbb{F}, \mathbb{D}, \mathbb{E})$ that represents the set of all constraints

- $\mathbb{A}$ is a finite set of possible atomic constraint sets $\left\{A_{c}\right\}$

- $\mathbb{F}$ is a finite set $\{$ unfair, 1-fair,fair\} of possible fairness constraints

- $\mathbb{D}$ is a set of quality constraints such as distance constraint $\delta_{t h} \geq 0$ or minimum SINR constraint

- $\mathbb{E}$ is a set of all balance constraints $\epsilon_{t h} \geq 0$. 
Table 3 Channel assignment problems.

\begin{tabular}{lll}
\hline $\mathbb{F}$ & $\mathbb{E}$ & Channel Assignment Problem \\
\hline unfair & - & unfair distance constrained assignment \\
1-fair & $|E|$ & 1-fair distance constrained assignment \\
fair & $|E|$ & fair distance constrained assignment \\
fair & $\epsilon_{t h}$ & fair, balanced and distance constrained assignment \\
\hline
\end{tabular}

The channel assignment algorithms (CAA) have two basic objectives:

- maximize the number of links in every sub-channel (maximized total link capacity),

- minimize the total number of sub-channels used subject to all the constraints (minimizes total delay).

The sub-channels we consider are the time slots in a time frame. Depending on fairness, distance and balance constraints we may classify various channel assignment problems as shown in Table 3 .

We will now formulate the two different objectives (as given in Definition 4) of a CAA. In doing so, we identify the correspondence of these problems to other well known problems to get an idea on the complexity. Henceforth, we call the problem of maximizing the number of links assigned to a given sub-channel as the sub-channel assignment problem and the problem of minimizing the number of sub-channels used in channel assignment as the sub-channel minimization problem.

\subsection{Sub-Channel Assignment Problem (SAP)}

Given a multi-hop wireless network $G=(V, E)$, if a subset of edges $\{e\} \subseteq E$ satisfies $A_{c}$ and $\delta_{t h}$, this set is called a transmission set [1]. A naive way to enumerate all the transmission sets for $G$ is to take all possible combinations of edges in $E$ and test to see if they satisfy the specified constraints. The complexity of this algorithm is

$$
\sum_{i=1}^{|E|}\left(\begin{array}{c}
|E| \\
i
\end{array}\right)
$$

which is exponential in $|E|$. The objective of SAP is to find out the largest possible transmission set for a given sub-channel.

Problem 1 The objective of SAP is to assign maximum number of links to a given sub-channel subject to the specified atomic and distance constraints $A_{c}$ and $\delta_{t h}$, respectively. 
To analyze the complexity of SAP, we compare it with the well known MaxClique problem [3]. Given is an instance of SAP, $(G, \mathfrak{c})$, where $\mathfrak{c} \in \mathfrak{C}$ is a set of given constraints. Algorithm SAP-to-MaxClique transforms the objective of SAP to that of finding maximum clique in a graph. Note that in the graph

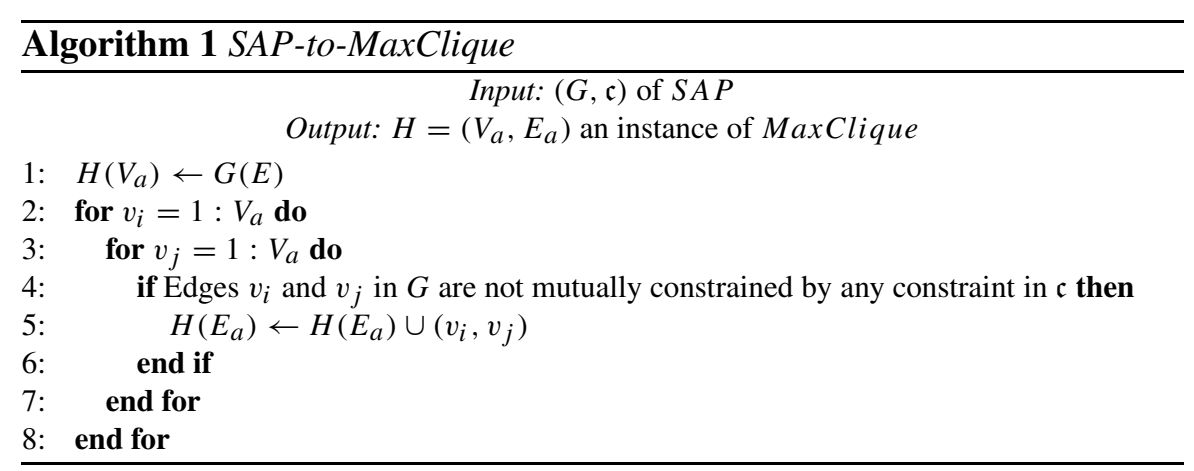

$H=\left(V_{a}, E_{a}\right)$, vertices of $H$ represent edges of $G$ and any two edges in $G$ that are mutually constrained in $\mathfrak{c} \in \mathfrak{C}$ do not have an edge in $H$ between the corresponding vertices.

The complexity of algorithm SAP-to-MaxClique is $\Theta\left(|E|^{2}\right)$, which is polynomial in $E$. As none of the vertices in any complete subgraph of $H$ are mutually constrained by any of the constraints specified by $\mathfrak{c}$, they all can be scheduled in the same sub-channel. This makes every complete subgraph of $H$ a transmission set of $G$. Hence the sub-channel allocation problem is to determine the maximum clique of $H$. The decision version of this problem is to determine whether a clique of a given size $J$ exists in the graph.

Problem 2 MaxClique decision problem.

Instance: A graph $H=(V, E)$ and a positive integer $J \leq|V|$.

Question: Does $H$ contain a clique of size $J$ or larger, that is, is there a subset $V^{\prime} \subseteq V$ such that $\left|V^{\prime}\right| \geq J$ and every two vertices in $V^{\prime}$ are joined by an edge in $E$ ?

If $J$ is not a constant significantly smaller than $|V|$, this problem and the corresponding optimization problem are known to be NP complete [15]. Note that a naive way to solve SAP is to enumerate all possible sub-graphs of $H$ and determine if the size of largest complete subgraph is $J$. The running time of the naive algorithm (Naive-MC) to solve the MaxClique is $\Omega\left(J^{2}\left(\begin{array}{c}|V| \\ J\end{array}\right)\right)$ ), which is polynomial if $J$ is a constant [3]. 


\subsection{Sub-Channel Minimization Problem (SMP)}

Given a multi-hop wireless network $G=(V, E)$, the number of transmission sets is upper bounded by (9). However, for given constraints $\mathfrak{c} \in \mathfrak{C}$ the number of feasible transmission sets may be reduced. The objective of the sub-channel minimization problem is to find a collection of minimum number of transmission sets such that every edge in the network appears in at least one transmission set.

Problem 3 The sub-channel minimization problem (SMP) is to find a 1-fair or fair assignment that minimizes the number of sub-channels, $T$, subject to the specified atomic, distance and balance constraints $A_{c}, \delta_{t h}, \epsilon_{t h}$ respectively. In other words, minimize T, such that $\bigcup_{t=1}^{T} E_{t}=E, A_{c},(2)$ and (8) hold.

To analyze the complexity of SMP we compare it with the minimum cover problem. Given a channel assignment problem, algorithm SMP-to-MinCover transforms this problem to a family of sets $\mathcal{F}$ such that any set $s \in \mathcal{F}$ is a transmission set. Then the objective of SMP can be seen to be the objective of finding a minimum cover for $\mathcal{F}$.

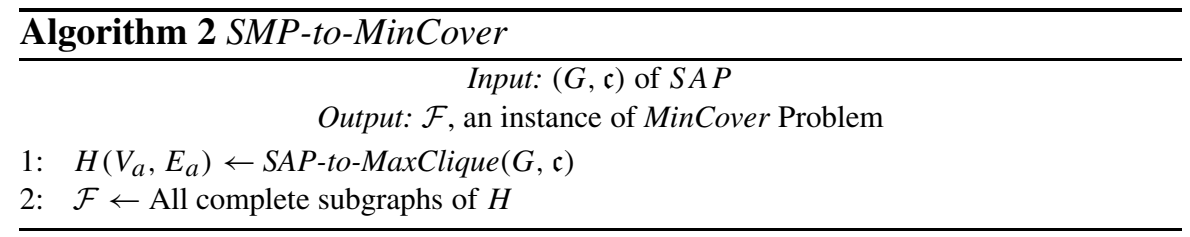

The complexity of algorithm SMP-to-MinCover is equivalent to that of $S A P$ with the same set of constraints. To solve $S M P$, we need to find a minimum cover of $\mathcal{F}$. The decision version of the minimum cover problem is to determine if a cover of size $T$ or less exists given a family of sets $\mathcal{F}$, where the cardinality of every set in $\mathcal{F}$ is upper bounded by $k$.

Problem 4 Minimum $k$-cover problem.

Instance: Family $\mathcal{F}$ of subsets over $E$ such that $\forall s \in \mathcal{F},|s| \leq k$, and a positive integer $T$.

Question: Does $\mathcal{F}$ contain a cover for $E$ of size $T$ or less, that is, is there a subset $\mathcal{F}^{\prime} \subseteq \mathcal{F}$ with $\left|\mathcal{F}^{\prime}\right| \leq T$ and such that $\bigcup_{e \in \mathcal{F}^{\prime}} e=E$ ?

This problem is proved to be NP complete in [15] by reducing it to the exact cover problem, which is a known NP complete problem. However, if 
the maximum cardinality of sets in $\mathcal{F}$ is two (i.e. $k=2$ ), then the problem of finding minimum cover can be solved in polynomial time.

\section{Complexity of Channel Assignment Problems}

In this section we analyze the complexity of the sub-channel assignment (further referred to as channel assignment) problems presented in Table 3. Note that all these problems belong to the class NP [15] in general. However, in the remaining of the section, for each of the channel assignment problems we either show that it can be solved in polynomial time or we prove that it is NP hard under certain conditions.

\subsection{Unfair and Distance Constrained Channel Assignment}

The unfair and distance constrained channel assignment problem can be stated as follows.

Problem 5 unfair- $\delta$ - $C A P$.

Given a channel assignment problem instance $(G, \mathfrak{c})$ with $\mathfrak{c}=\left(A_{c}\right.$, unfair, $\left.\delta_{t h},|E|\right)$ and $k$, a positive integer, is there a solution to unfair-CAP of size $k$ i.e., is there a subset $E_{t} \subseteq E$ with $\left|E_{t}\right| \geq k$ such that all the constraints in $\mathfrak{c}$ are satisfied?

To determine the complexity of solving this problem, we explore the implication of distance constraint on NP completeness of the unfair channel assignment problem. Our first step in this direction is to arrive at an upper bound on the number of simultaneous transmissions that are possible for a wireless network spread over a geographical area $A$ with distance constraint $\delta_{t h}$ and maximum transmission radius $R_{\max }$. It can shown following the work in [5] that under the protocol model the number of simultaneous transmissions $J$ is upper bounded by $\frac{4 c}{\pi \delta_{t h}^{2} r_{\max }^{2}}$, where $0<c<1$ is a suitable constant and $r_{\max }=\frac{R_{\max }}{\sqrt{A}}$ is the normalized maximum transmission radius.

Theorem 1 For a given distance constraint $\delta_{t h}$, radius $r_{\max }$, and area $A$ of the wireless network, the unfair channel assignment problem can be solved in polynomial time, if $|E| \gg J$.

Proof. To show that this problem is polynomial time solvable, we propose an algorithm unfair- $\delta$-CAP that solves every instance of the given problem and prove that the algorithm runs in polynomial time. 


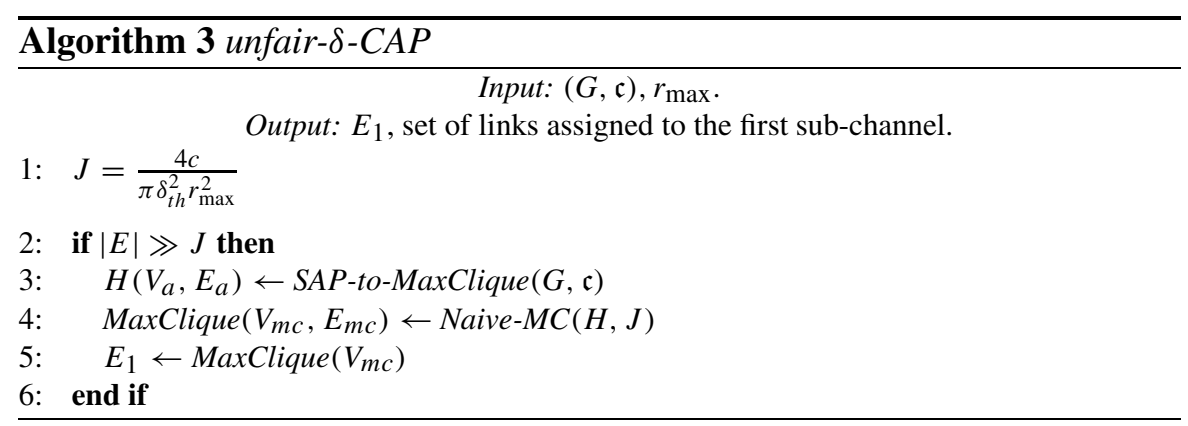

We know that SAP-to-MaxClique $(G)$ runs in $\Theta\left(|E|^{2}\right)$. Note that $J \ll|E|$ here is a constant independent of $E$ which implies that $J$ remains constant with respect to an increase in the number of edges $E$. Using this fact we can compute the complexity of Naive-MC algorithm as

$$
\begin{aligned}
\text { Naive-MC(G,J) } & \cong \Theta\left(\sum_{i=1}^{J}\left(\begin{array}{c}
|E| \\
i
\end{array}\right)\right) \\
& \cong \Theta\left(|E|^{J}\right), \forall J \ll|E|
\end{aligned}
$$

The complexity of unfair- $\delta$-CAP algorithm is $\mathcal{O}\left(|E|^{J}\right)$, which is polynomial in $|E|$.

\subsection{1-Fair and Distance Constrained Channel Assignment}

The 1-fair distance constrained channel assignment is defined as follows:

Problem 6 1-fair- $\delta$-CAP.

Instance: A channel assignment problem instance $(G, \mathfrak{c})$ with $\mathfrak{c}=$ $\left\{A_{c}, \delta_{t h},|E|\right\}$ such that $|E| \gg J$ a positive integer $T$, a set of positive integers $\left\{k_{1}, \ldots, k_{T}\right\}$.

Question: Is there an 1-fair- $\delta$ constrained channel assignment for $G$ with total number of sub-channels lesser or equal to $T$ and each sub-channel of size $k$ ? That is, is there a channel assignment $\left\{E_{t}\right\}$, with $\left|\left\{E_{t}\right\}\right| \leq T$ and $\forall E_{t_{i}} \in\left\{E_{t}\right\},\left|E_{t_{i}}\right| \geq k_{i}$ such that all the constraints in $\mathfrak{c}$ are satisfied ?

In the following lemma we show a special class of 1-fair- $\delta$-CAP for which, every input instance of the 1 -fair- $\delta-C A P$ can be represented as a family $\mathcal{F}$ 
of feasible transmission sets with the cardinality of $\mathcal{F}$ polynomial in $|E|$. In the related corollary we show that the above transformation of 1-fair- $\delta$-CAP input instance to family $\mathcal{F}$ can be done in polynomial time.

Lemma 1 Given an instance of 1-fair- $\delta$-CAP in a wireless network with area $A$, maximum radius $r_{\max }$ and constraint $\delta_{t h}$, the number of feasible transmission sets is polynomial in $|E|$ if $|E| \gg J$.

Proof. Follows from Theorem 1.

Corollary 1 Transformation $\pi: 1$-fair- $\delta$-CAP $\rightarrow \mathcal{F}$ can be done in polynomial time for a given wireless network with area $A$, maximum radius $r_{\max }$ and constraint $\delta_{\text {th }}$ when $|E| \gg J$.

Proof. Follows from Theorem 1, using Naive-MC algorithm.

The optimal channel assignment $\left\{E_{t}\right\}$ of 1 -fair- $\delta$-CAP is a collection of transmission sets. Since $\mathcal{F}$ is the set of all feasible transmission sets given the constraints $\mathfrak{c}$, we have $\left\{E_{t}\right\} \subseteq \mathcal{F}$.

Lemma 2 If two sets $s_{i}, s_{j} \in \mathcal{F}$ s.t $s_{i} \subset s_{j}$ then $s_{i} \notin\left\{E_{t}\right\}$, where $\left\{E_{t}\right\}$ is the optimal 1-fair- $\delta$-CAP solution.

Proof. Assume $s_{i} \in\left\{E_{t}\right\}$. This implies $s_{j} \in\left\{E_{t}\right\}$, since 1-fair- $\delta$-CAP outputs sets with maximum cardinality. Since $\left\{E_{t}\right\}$ is optimal, $s_{i} \notin\left\{E_{t}\right\}$, a contradiction.

We can hence represent the family $\mathcal{F}$ by its maximal feasible sets $\mathcal{F}^{\text {max }}$ such that it is not possible to have two sets $s_{i}, s_{j}$ such that $s_{i} \subset s_{j}$; and $s_{i}, s_{j} \in \mathcal{F}^{\max }$. Note that any optimal solution on $\mathcal{F}$ will be completely contained in $\mathcal{F}^{\text {max }}$. However the use of $\mathcal{F}^{\text {max }}$ instead of $\mathcal{F}$ may reduce the search complexity.

Theorem 2 1-fair- $\delta$-CAP is NP-complete.

Proof. The maximum cardinality of a transmission set in the family $\mathcal{F}^{\text {max }}$ can be upper bounded by $J$ (from Lemma 1). The objective of 1 -fair- $\delta$-CAP will then be to pick a subset $f \subseteq \mathcal{F}$ such that every edge in $E$ is covered by $f$. For $k=J$ and $\left\{E_{t}\right\}=f$, the well known $k$-Set Cover problem (see Definition4) and 1-fair- $\delta$-CAP are equivalent (from Lemma 1 and Corollary 1). Hence for $J \geq 3$ this problem is NP complete and for $J \leq 2$ optimal solutions can be found in polynomial time using matching techniques $[8,9]$. 


\subsection{Fair Distance Constrained Channel Assignment}

The fair distance constrained channel assignment can be defined as follows.

Problem 7 fair- $\delta$ - $C A P$.

Instance: A channel assignment problem instance $(G, \mathfrak{c})$ with $\mathfrak{c}=$ $\left\{A_{c}\right.$, fair, $\left.\delta_{t h},|E|\right\}$ such that $|E| \gg J$ a positive integer $T$, a set of positive integers $K=\left\{k_{1}, \ldots, k_{T}\right\}$.

Question: Is there an fair- $\delta$-CAP for $G$ with number of sub-channels lesser or equal to $T$ and each sub-channel of size $K$ ? That is, is there a channel assignment $\left\{E_{t}\right\}$, with $\left|\left\{E_{t}\right\}\right| \leq T, \forall E_{t_{i}}, E_{t_{j}} \in\left\{E_{t}\right\}, E_{t_{i}} \cap E_{t_{j}}=\phi$ with $\left|E_{t_{i}}\right| \geq k_{i}$ such that all other constraints in $\mathfrak{c}$ are satisfied?

From Lemma 1 and Corollary 1 it follows that every input instance of fair$\delta$ - $C A P$ can be represented as a family $\mathcal{F}$ of feasible transmission sets in polynomial time. The objective of $f a i r-\delta-C A P$ is then to find a minimal subcover $f$ which has no overlap. This minimal subcover $f$ will form the optimal channel assignment $\left\{E_{t}\right\}$ satisfying all the constraints in $\mathfrak{c}$.

Theorem 3 fair- $\delta$-CAP is NP complete.

Proof. For $k=J$ and $\left\{E_{t}\right\}=f$, the SET COVERING II problem [8] is equivalent to fair- $\delta$-CAP. Hence, for $J \geq 3$ this problem is NP complete and for $J \leq 2$ optimal solutions can be found in polynomial time.

\subsection{Fair Distance Constrained and Balanced Channel Assignment}

The fair distance constrained and balanced channel assignment is defined as follows:

Problem 8 fair- $\delta$-Balanced-CAP.

Instance: A channel assignment problem instances $(G, \mathfrak{c})$ with $\mathfrak{c}=$ $\left\{A_{c}\right.$, fair $\left., \delta_{t h}, \epsilon_{t h}\right\}$ such that $|E| \gg J$ a positive integer $T$, a set of positive integers $\left\{k_{1}, \ldots, k_{T}\right\}$.

Question: Is there fair- $\delta$-Balanced-CAP for $G$ with number of sub-channels lesser or equal to $T$ and each sub-channel of size $k$ ? That is, is there a channel assignment $\left\{E_{t}\right\}$, with $\left|\left\{E_{t}\right\}\right| \leq T$ and $\forall E_{t_{i}}, E_{t_{j}} \in\left\{E_{t}\right\}, E_{t_{i}} \cap E_{t_{j}}=\phi$ with $\left|E_{t_{i}}\right| \geq k_{i}$ and $\epsilon_{t h}$ balanced such that all other constraints in $\mathfrak{c}$ are satisfied?

Theorem 4 fair- $\delta$-Balanced-CAP is NP complete. 
Proof. Restrict to partition problem [15] by having $T=2, \epsilon_{t h}=0$ and $\forall e_{i}, s\left(e_{i}\right)=1$.

\section{Algorithms to Solve Distance Constrained Unfair, 1-fair and fair CAP}

We have seen that algorithm SMP-to-MinCover transforms any given instance of channel assignment problem into a set $\mathcal{F}$ of subsets $\left\{E_{t}\right\}$ of the edges $E$ of the wireless network. In this section we propose another algorithm called Gen- $\mathcal{F}^{\max }$ which is similar to SMP-to-MinCover but with a huge reduction in complexity. Here, instead of generating the entire family $\mathcal{F}$, we generate only the maximal family $\mathcal{F}^{\text {max }}$ using a novel tree pruning approach. For any given $C A P$ instance, $G e n-\mathcal{F}^{\text {max }}$ constructs the corresponding maximal family $\mathcal{F}^{\max }$.

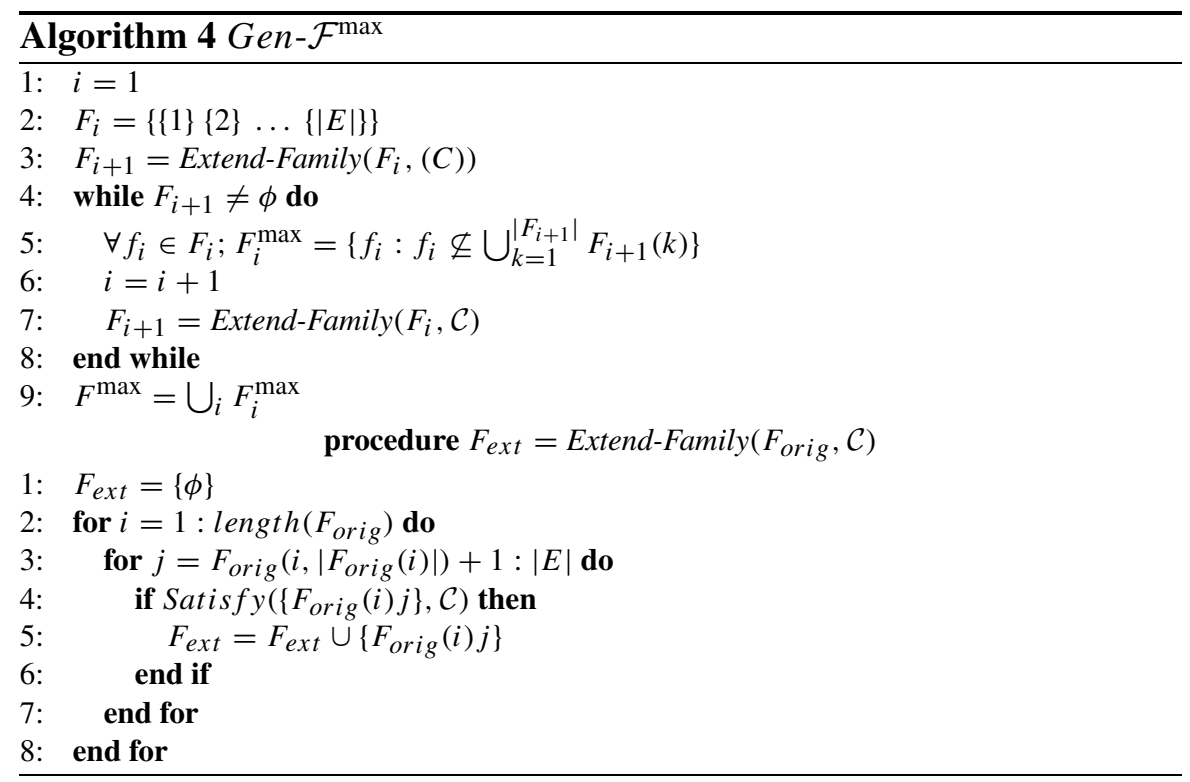

An instance of $G e n-\mathcal{F}^{\max }$ on a six edge example network (Figure 4) is shown in Figure 5. Initially every edge in the family is allocated to a singleton set. Thus the initial family comprises of as many subsets as the number of edges. For the given example, the initial family is $\{\{1\}\{2\}\{3\}\{4\}\{5\}\{6\}$ $\{7\}$ \}. In the next and the following rounds two fundamental operations take place. The first operation is expansion, where each of the subsets in the family 


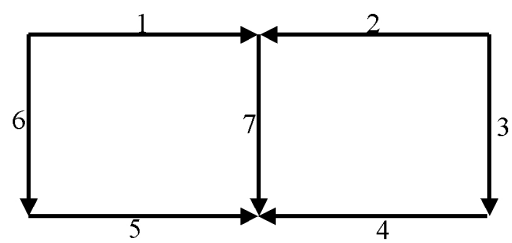

Figure 4 Example network with six edges.

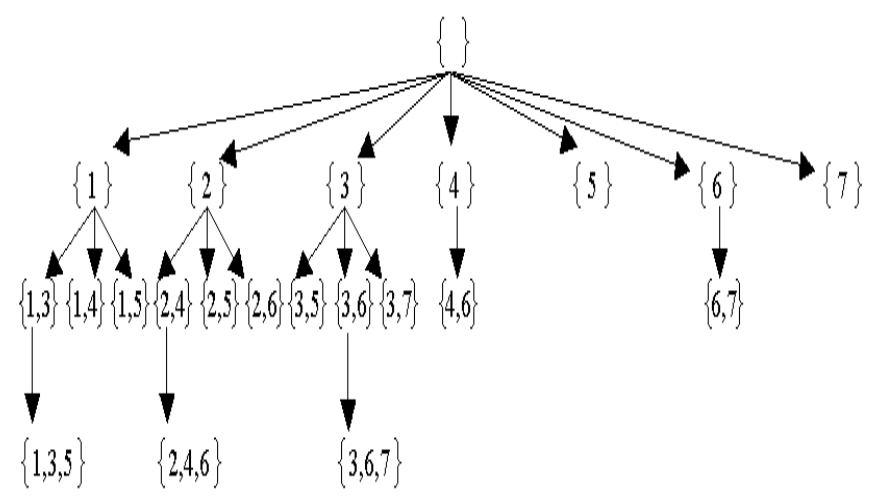

Figure 5 Instance of $G e n-\mathcal{F}^{\max }$ on a six edge example network.

is expanded by including one more unique edge. For the given example, the family obtained after first expansion is $\{\{1,3\}\{1,4\}\{1,5\}\{2,4\}\{2,5\}\{2,6\}$ $\{3,5\}\{3,6\}\{3,7\}\{4,6\}\{6,7\}\{1\}\{2\}\{3\}\{4\}\{5\}\{6\}\{7\}\}$. Here, we do not see $\{1,2\},\{1,6\},\{2,3\}$.etc as they do not satisfy the constraint set. The second operation is pruning, where the subsets which are in-turn subsets of larger sets are eliminated from the family. For the given example, the family obtained after first pruning is $\{\{1,3\}\{1,4\}\{1,5\}\{2,4\}\{2,5\}\{2,6\}\{3,5\}$ $\{3,6\}\{3,7\}\{4,6\}\{6,7\}\}$. These two operations are performed iteratively until expansion stops. For the given example, the final family is $\{\{1,3,5\}$ $\{2,4,6\}\{3,6,7\}\}$.

Theorem 5 For any given instance of $C A P$, Algorithm Gen- $\mathcal{F}^{\max }$ constructs a maximal family $F^{\max }$.

Proof. Follows by induction on $\bigcup_{1}^{i} \mathcal{F}_{i}$.

The following lemma and theorem establish that the proposed pruning algorithm has polynomial order complexity. 
Lemma 3 The probability $\alpha$ of co-existence of a pair of links is $1-\pi \delta_{t h}^{2} r_{\max }^{2}$.

Proof. The probability of co-existence of any pair of links in the network is given by the probability that the receiving node of the second link is at least $\delta_{t h} r_{\max }$ apart from the receiving node of the first link (see Figure 3). That is, the probability the second receiving node being outside the circle with radius $\delta_{t h} r_{\max }$ centered around the first receiving node. Clearly, this probability is equal to $\alpha=1-\pi \delta_{t h}^{2} r_{\max }^{2}$.

Theorem 6 The expected number of feasible transmission sets is upper bounded by $\left(|E|-\pi \delta^{2} R_{\max }^{2} n_{o}\right)^{J}$, where $J=\frac{4 c}{\pi \delta_{t h}^{2} r_{\max }^{2}}, r_{\max }=R_{\max } / \sqrt{A}$ and $n_{o}$ is the edge density in the network.

Proof. The expected number of pairs of links in the network is $\alpha\left(\begin{array}{l}n \\ 2\end{array}\right)$, where $n=|E|$. For any transmission set of cardinality $k$ with $k>1$, the

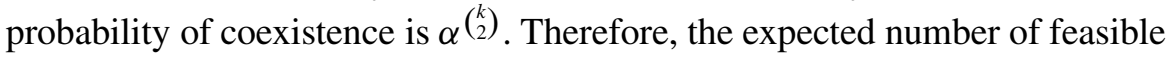
transmission sets is given by

$$
\begin{aligned}
& E(|\mathcal{F}|)=\sum_{k=2}^{J} \alpha^{\left(\begin{array}{l}
k \\
2
\end{array}\right)}\left(\begin{array}{l}
n \\
k
\end{array}\right) \\
& =\sum_{k=2}^{J} \alpha^{\frac{k(k-1)}{2}} \frac{n !}{(n-k) ! k !} \\
& \leq \sum_{k=2}^{J} \alpha^{k^{2}} n^{k} \\
& \leq \sum_{k=0}^{J}(\alpha n)^{k}
\end{aligned}
$$

if $(\alpha n)>1, E(|\mathcal{F}|) \cong \mathcal{O}\left(\frac{(\alpha n)^{J+1}-1}{(\alpha n)-1}\right)$

if $(\alpha n)<1, E(|\mathcal{F}|) \cong \mathcal{O}\left(\frac{1}{1-(\alpha n)}\right)$

if $(\alpha n)=1, E(|\mathcal{F}|) \cong \mathcal{O}(J)$.

The edge density of the network is given by $n_{o}=|E| / A$. Hence from the above three cases we see that the upper bound of $E(|\mathcal{F}|)$ is $\mathcal{O}\left(\frac{(\alpha n)^{J+1}-1}{(\alpha n)-1}\right) \cong \mathcal{O}(\alpha n)^{J}=\mathcal{O}\left(|E|-\pi \delta^{2} R_{\max }^{2} n_{o}\right)^{J}$.

Since Gen- $\mathcal{F}^{\max }$ looks only at the feasible transmission sets, the running time of $G e n-\mathcal{F}^{\max }$ is $\mathcal{O}(E(|\mathcal{F}|))$. 


\subsection{Optimal unfair-CAA}

An optimal unfair channel assignment algorithm is the following. Choose the set with the largest cardinality in $\mathcal{F}^{\text {max }}$ generated by $G e n-\mathcal{F}^{\text {max }}$. The solution is optimal because, Gen- $\mathcal{F}^{\text {max }}$ generates the family of all possible large transmission sets. That is, there cannot be a transmission that is larger than the all of the transmission sets in $\mathcal{F}^{\text {max }}$ and satisfy all the specified constraints.

When $|E| \gg J$ this algorithm produces optimal solution for unfair-CAP and the running time is same as the Gen- $\mathcal{F}^{\text {max }}$ algorithm.

\subsection{Approximate 1-fair-CAA}

We know that there can be no deterministic algorithm that solves 1-fair-CAP in polynomial time. Heuristic algorithms [8] that solve minimum cover problem can be used on 1-fair-CAP. The input instances of heuristic algorithms that solve minimum cover problem should be mapped from instances of $\left\{\mathcal{F}^{\text {max }}, E\right\}$ of 1-fair-CAP and the minimum cover output instances should be mapped to channel assignment of 1-fair-CAP.

We use the greedy approximation algorithm discussed in [3]. This algorithm works in a greedy fashion selecting the set that covers the maximum number of uncovered elements in every iteration. The run time complexity of this algorithm is $\mathcal{O}\left(\sum_{s \in \mathcal{F} \text { max }}|s|\right)$. It is shown that this algorithm returns a set cover that has an approximation ratio bound of $H\left(\max \left\{|s|: s \in \mathcal{F}^{\max }\right\}\right)$ over the optimal solution, here $H(d)$ denotes the $d^{\text {th }}$ harmonic number $H(d)=\sum_{i=1}^{d} 1 / i$. From Section 5.1 we know that $\max \left\{|s|: s \in \mathcal{F}^{\max }\right\} \leq J$ where $J$ is independent of $E$. Hence the approximation ratio of greedy set cover for this case is $H(J)$.

\subsection{Approximate fair-CAA}

By Theorem 5.3 fair distance constrained channel assignment problem is NP complete. The greedy heuristic based on graph coloring [13] can be used directly for the $\delta_{t h}=0$ case. However, for distance constrained assignment a slight modification to the unified algorithm given in [13] generates fair channel assignment satisfying the distance constraints. 


\section{Simulation Results}

\subsection{Performance Measures}

To evaluate the effectiveness of the channel assignment algorithms proposed in Section 6, we use the following performance measures.

\subsubsection{Number of Sub-Channels}

The total number of sub-channels (denoted by $\mathrm{T}$ ) used by the assignment technique is an important performance metric. In any protocol, there is some overhead associated with each sub-channel and this overhead adds up for every new sub-channel. When the sub-channels are in the form of time slots, then more number of time slots directly translates to increased delay. Hence it is generally preferable to have a schedule that uses least number of subchannels while maintaining high link capacities.

\subsubsection{Capacity}

We use two different notions of capacity. The first one is protocol capacity, which is the average number of links scheduled to transmit per sub-channel. That is,

$$
C=\frac{1}{T} \sum_{t=1}^{T}\left|E_{t}\right|
$$

The second notion for capacity is the sum rate capacity, which is the sum of Shannon's capacity [11] for each link divided by the total number of subchannels used.

\subsubsection{Imbalance}

Imbalance is the maximum absolute difference in the number of links between two sub-channels.

\subsection{Performance of Proposed Channel Assignment Algorithms}

We evaluate the performance of the unfair, 1-fair and fair channel assignment algorithms for wireless networks with varying node densities. We use both the grid network model and the random network model. The area of the network used in the simulation is $100 \times 100$ square meters.

For the grid networks, we fix 16 nodes on the network. Initially we do not assume any distance ratio constraint. We then evaluate both the protocol and the sum rate capacity for Unfair and 1-fair channel assignment. The same 


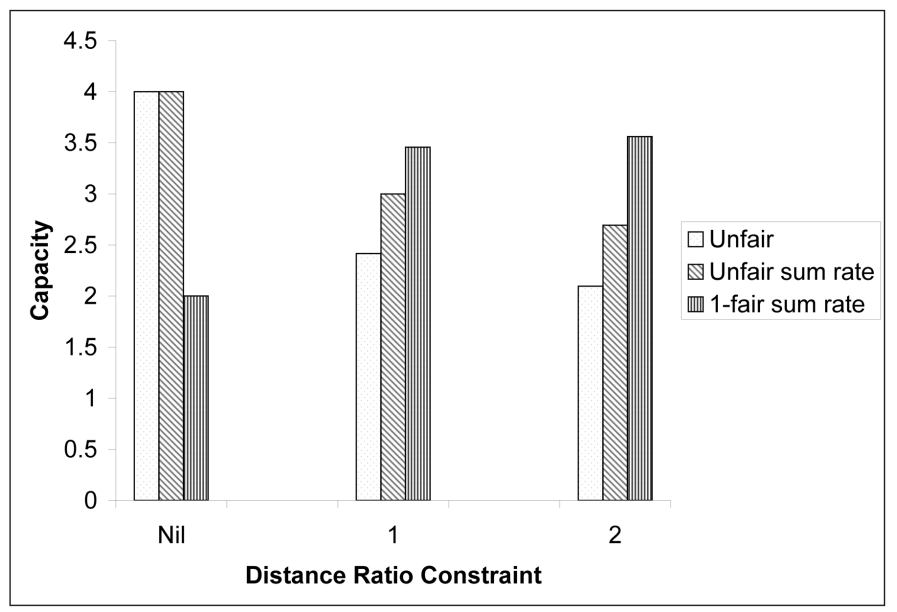

Figure 6 Capacity vs. distance ratio constraint for a grid network with area of $100 \times 100$ square meters.

experiment is performed for distance ratio constraints with $\delta$ equal to 1 and 2. The corresponding results are plotted in Figure 6. Note that Nil represents the absence of distance ratio constraint. We can observe from the figure that as the distance ratio constraint increases the capacity defined by protocol model seems to decrease where as the sum rate capacity, which is the true capacity of the network increases. We can further observe that there is a gain of about $70 \%$ in sum rate capacity in 1-fair channel assignment when distance ratio constraint is introduced. This suggests that SINR/distance constrained protocols result in better channel assignments in terms of sum rate capacity.

For the random network model, we fixed the distance ratio constraint $\delta_{t h}$ to 3 . The maximum transmission range of the network is fixed to 10 meters. Figure 7 shows the comparison of the capacities of unfair, 1-fair and fair channel assignment algorithms as a function of average number of edges in the network. We can see that as the fairness constraint is relaxed from exact fairness to unfair the capacity achieved increases. For example, Figure 7 shows that for a network with 80 edges, there is a $100 \%$ gain in capacity when the fairness is relaxed from exact fairness to 1 -fairness and $25 \%$ gain when fairness constraint is relaxed from 1-fairness to unfair. The number of subchannels used to cover the network by 1-fair and fair are given in Figure 8. We can observe that the heuristic based on covering and coloring result in 


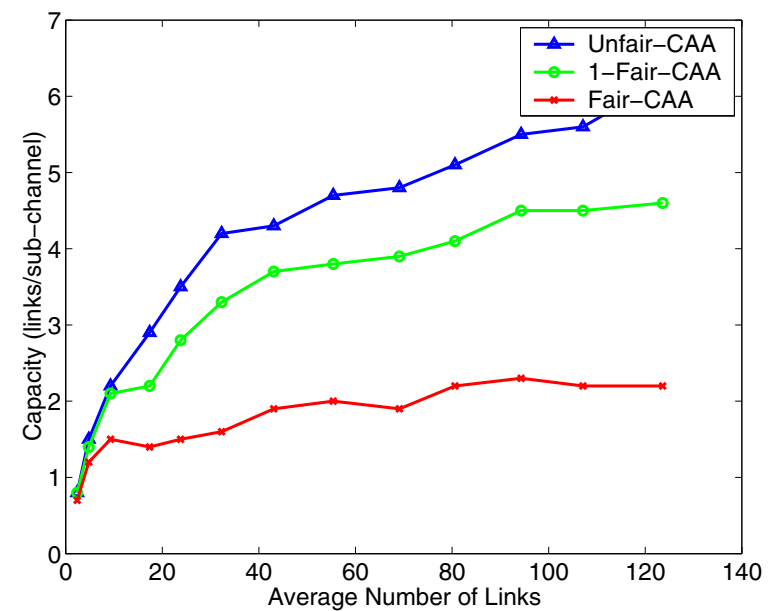

Figure 7 Performance of unfair, 1-fair, and fair CAA in terms of average number of links per sub-channel versus the number of edges, in a network with area $100 \times 100$ square meters, distance constraint $\delta_{t h}=3$ and maximum range $=10$ meters.

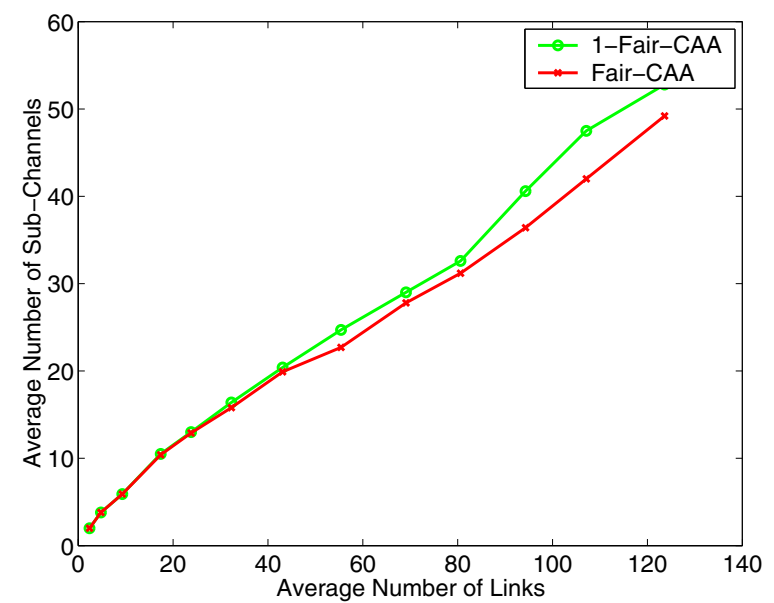

Figure 8 Performance of 1-fair and fair CAA in terms of the number of sub-channels used versus the number of edges, in a network with area $100 \times 100$ square meters, distance constraint $\delta_{t h}=3$ and maximum range is 10 meters. 


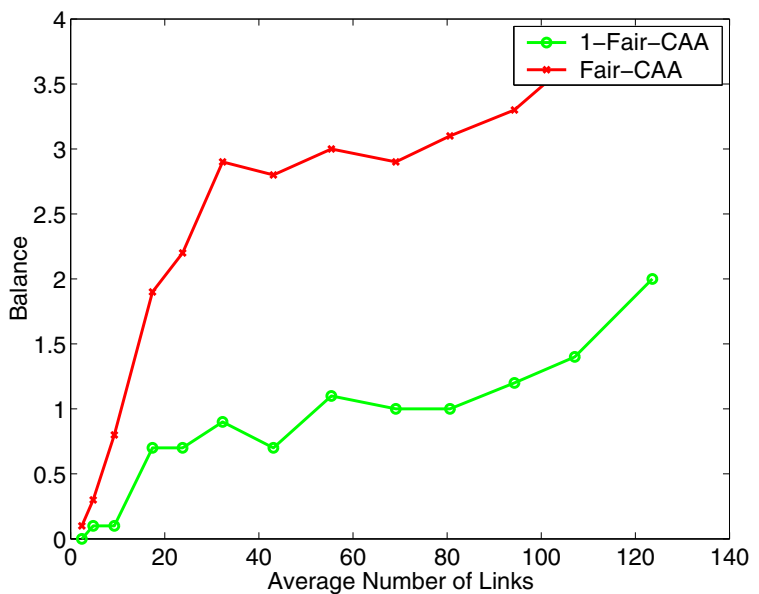

Figure 9 Performance of 1-fair and fair CAA in terms of balance versus the number of edges, in a network with area $100 \times 100$ square meters, distance constraint $\delta_{t h}=3$ and maximum range is 10 meters.

comparable performance over all networks considered. The imbalance of 1-fair and fair channel assignments over different sub-channels is given in Figure 9. Here we can see that the maximum value of imbalance for 1-fair $C A A$ is equal to 2 . This indicates that all the sub-channels have almost the same number of edges.

To study the impact of distance constraint $\delta_{t h}$ on the capacity of the channel assignment, we fix the number of edges in the network to 24 and vary the distance constraint from 0 to 10 . Figure 10 plots the reduction in capacity due to increase in the distance constraint $\left(\delta_{t h}\right)$. Here we can observe that for smaller distance constraints 1-fair and unfair CAA perform comparably, however as the distance constraint is increased 1-fair CAA performs poorly compared to unfair CAA. We can also observe that exact fair channel assignment quickly converges to the one link per sub-channel worst case behavior compared to 1-fair and unfair assignments.

We also study the capacity of the assignment for networks with different maximum transmission range, $R_{\max }$. We fix $\delta_{t h}=3$, number of nodes to 30 and vary the maximum allowable transmission range from 1 to 20 meters. Figure 11 plots the capacity versus increase in the maximum allowable range. We can observe that the capacities of channel assignments increase up to a point and then decreases. This is because at lower transmission range, the network has very few edges and many disconnected nodes. As the transmission 


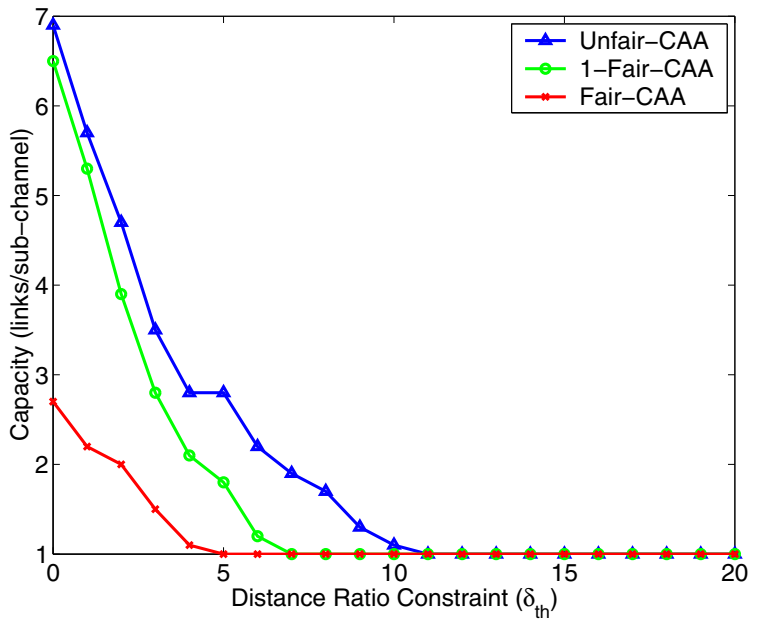

Figure 10 Performance of unfair, 1-fair, and fair CAA in terms of average number of links per sub-channel versus the distance constraint $\delta_{t h}$, in a network with 24 edges, area $100 \times 100$ square meters and maximum range is 10 meters.

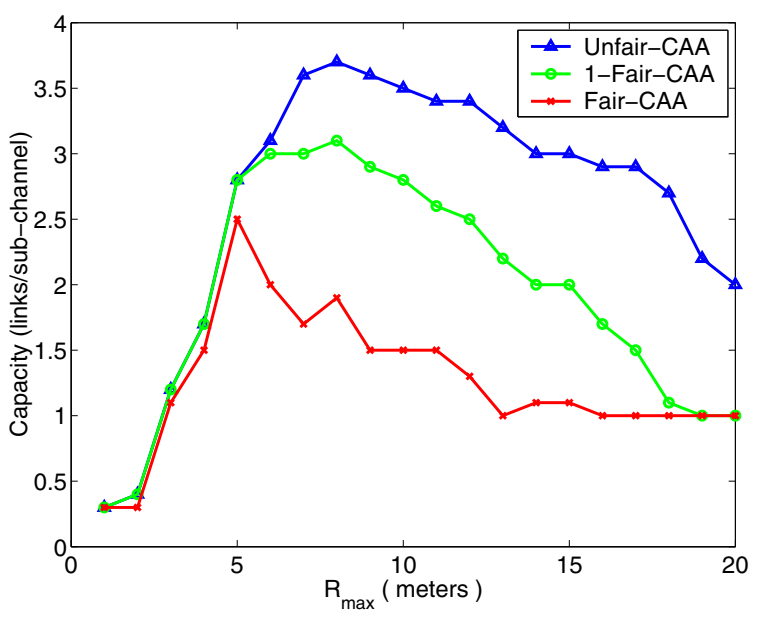

Figure 11 Performance of unfair, 1-fair, and fair CAA in terms of average number of links per sub-channel versus the maximum range, in a network with 30 nodes, area $100 \times 100$ square meters and $\delta_{t h}=3$. 
range increases the number of edges and hence the capacity also increases and reaches the optimum. The decrease in capacity can be explained by the bound $J$ where $r_{\max }$ appears in the denominator.

\section{Conclusion}

In this paper we systematically studied the channel assignment problem in presence of fairness, SINR and balance constraints. Both grid and random models of wireless network were used. Several channel assignment problems based on specific constraints were identified. We showed that a class of unfair channel assignment problems can be optimally solved in polynomial time. We also showed that with fair and 1-fair constraints the channel assignment problem remains NP complete. Optimal unfair channel assignment algorithm, approximate 1-fair channel assignment algorithm and approximate fair channel assignment algorithm were proposed. Simulation results revealed that there is a gain in capacity of $100 \%$ when fairness constraint is relaxed from exact fairness to 1-fairness and $25 \%$ when relaxed from 1-fairness to unfair. It was observed that for smaller values of the distance constraint 1-fair and unfair perform comparably. We also saw that 1 -fair channel assignment was more balanced than fair channel assignment.

\section{Acknowledgement}

This work was supported by NSF \#0916180 and NSF \#0917008.

\section{References}

[1] E. Arikan. Some complexity results about packet radio networks. IEEE Transactions on Information Theory, 30(4):681-685, July 1984.

[2] I. Chlamtac and A. Lerner. Fair algorithms for maximal link activation in multihop radio networks. IEEE Transactions on Communications, COM-35(7):739-746, July 1987.

[3] T. Cormen, C. Leiserson, R. Rivest, and C. Stein. Introduction to Algorithms. McGrawHill, second edition, 2002.

[4] A. Ephremides and T. Truong. Scheduling broadcasts in multihop radio networks. IEEE Transactions on Communications, 38(4):456-460, April 1990.

[5] P. Gupta and P. Kumar. The capacity of wireless networks. IEEE Transactions on Information Theory, 46(2):388-404, March 2000.

[6] B. Hajek and G. Sasaki. Link scheduling in polynomial time. IEEE Transactions on Information Theory, 34(5):910-917, September 1988. 
[7] J. L. Hammond and H. B. Russell. Properties of a transmission assignment algorithm for multi-hop packet radio networks. IEEE Transactions on Wireless Communications, 3(4):1048-1052, July 2004.

[8] D.S. Johnson. Approximation algorithms for combinatorial problems. In STOC'73: Proceedings of the Fifth Annual ACM Symposium on Theory of Computing, pages 38-49. ACM Press, New York, 1973.

[9] R. Karp. Reducibility among combinatorial problems. In R.E. Miller and J.W. Thatcher (Eds.), Compexity of Computer Computations, pages 85-103. Plenum Press, New York, 1972.

[10] S. Kutten and I. Chlamtac. A spatial reuse tdma.fddma for mobile multi-hop radio networks. In INFOCOM, 1985.

[11] A. Leon-Garcia and I. Widjaja. Communication Networks: Fundamental Concepts and Key Architectures. McGraw-Hill School Education Group, 1999.

[12] C.G. Prohazka. Decoupling link scheduling constraints in multi-hop packet radio networks. IEEE Trans. Comput., 38(3):455-458, 1989.

[13] S. Ramanathan. A unified framework and algorithm for channel assignment in wireless networks. Wireless Networks, 5:81-94, 1999.

[14] R. Ramaswami and K. Parhi. Distributed scheduling for broadcasts in a radio network. In INFOCOM, 1989.

[15] M.R.Garey and D.S. Johnson. Computers and Intractability, A Guide to the Theory of NP-Completeness. Freeman and Company, New York, 2003.

[16] P. Värbrand, D. Yuan, and P. Bjorklund. Resource optimization of spatial TDMA in ad hoc radio networks: A column generation approach. In INFOCOM, 2003.

\section{Biographies}

Chetan Mathur received his Ph.D. in Computer Engineering at Stevens Institute of Technology, New Jersey, USA in 2007. He has an MS in Computer Engineering from Stevens Institute of Technology, New Jersey, USA in 2003. Part of Chetan's MS thesis was patented by Stevens Institute of Technology. He was born in Bangalore, India in 1981. He received his BE degree in Computer Science from Visveshwaraiah Institute of Technology, Bangalore, India in 2002. Chetan has published several research papers in the fields of Cryptography, Coding theory and Dynamic spectrum access. He has also received numerous awards including the IEEE best student paper award presented at IEEE Consumer Communications and Networking Conference (CCNC 2006) and the IEEE student travel grant award presented at International Conference on Communications (ICC 2005). He is currently employed in the financial industry.

M.A. Haleem graduated from Stevens Institute of Technology with a Ph.D. in Electrical and Computer Engineering. His research interests are in the 
areas of wireless communications and signal processing. He is currently an Assistant Professor at KFUPM, Saudi Arabia.

K.P. (Suba) Subbalakshmi is an Associate Professor at Stevens Institute of Technology. Her research interests are in the areas of cognitive radio networks, wireless network security, media forensics as well as social networks. She is the Vice-Chair North America region of IEEE Technical Committee on Cognitive Networks. She has given several key-note addresses, plenary talks and tutorials on DSA security at several international conferences. She has also served as a panelist on cognitive radio network security at international conferences including IEEE Dynamic Spectrum Access: Collaboration between the Technical, Regulatory and Business Communities, IEEE ICC, IEEE Sarnoff Symposium etc. She was a Guest Editor of the EURASIP Journal on Advances in Signal Processing, Special Issue on Dynamic Spectrum Access for Wireless Networks. Her work is/has been supported by the National Science Foundation, National Institute of Justice, DoD agencies as well as the Industry. Suba is also the co-founder of two companies that seek to commercialize some of her research work. One of these is Dynamic Spectrum LLC which commercializes her work in Dynamic Spectrum Access Networks.

R. Chandramouli (Mouli) is the Thomas Hattrick Chair Professor of Information Systems in the Department of Electrical and Computer Engineering (ECE) at Stevens Institute of Technology and Co-Director of the Information Networks and Security (iFINITY) research laboratory. He is a Co-Founder of Dynamic Spectrum, LLC - a startup offering cloud-enabled cognitive radio technologies for various markets including consumer communications, public safety, and the DoD; and Jaasuz.com that provides a suite of advanced text forensics technologies to verify trust in documents. His research spans the areas of wireless networking, social media analytics/security and computational psycho-linguistic text mining. 